\title{
Knowledge and attitude toward hearing impairment and vestibular disorders among medical students of north India
}

\author{
Himanshu Kumar Sanju ${ }^{1 *}$, Komal Aggarwal ${ }^{2}$, Arun Kumar Yadav ${ }^{3}$ \\ ${ }^{1,3}$ Assistant Professor, ${ }^{2}$ Audiologist, ${ }^{1}$ Dept. of ENT and Audiology, ${ }^{2,3}$ Dept. of Audiology, ${ }^{\mathbf{1}}$ Shri Jagdamba Charitable Eye Hospital, Sri \\ Ganganagar, Rajasthan, ${ }^{1}$ LKC Shri Jagdamba Andh Vidyalaya Samiti, Sri Ganganagar, Rajasthan, ${ }^{2,3}$ Amity Medical School, Amity \\ University, Haryana, India
}

\section{*Corresponding Author: Himanshu Kumar Sanju}

Email: himanshusanjuaslp@gmail.com

\begin{abstract}
Research studies have indicated that there is lack of awareness regarding the adverse effects of hearing loss and vestibular disorder among health professionals and upcoming medical students. So, there is a need to upgrade these professionals and train them which will turn result in proper referrals and better implementation hearing related programs in a health care set-up. Audiologists play a vital role in spreading awareness regarding their role and scope of practice, so they need to initially know the status and level of awareness and knowledge regarding hearing loss and vestibular disorder among the medical students. The present research is being conducted to assess the knowledge and attitude among medical students across various range of hospital set up in northern India regarding hearing loss, vestibular disorder \& assessment procedures available. As, these medical students are going to become a qualified physician, henceforth its very necessary that the students have the basic knowledge and must be aware regarding the role of an audiologist/speech language pathologist. A questionnaire-based study (online survey) of 22 questions was adopted from similar kind of studies and was framed with the aim to assess the knowledge and attitude among allied health professionals regrading hearing loss and vestibular disorder. A total of 226 medical students from government and private medical colleges spread across Northern India participated in the present study. The outcome of the present study reveals positive result indicating that participants had good knowledge and attitude towards the basic areas related to hearing loss and vestibular disorders. However, there some drawbacks in certain areas which has be corrected with proper training and knowledge.
\end{abstract}

Keywords: Knowledge, Attitude, Hearing Impairment, Vestibular Disorder, Medical Students.

\section{Introduction}

Hearing is one of the most important primary senses which help us to communicate smoothly with the hearing world. Unfortunately, the sense of hearing is often being kept neglected and people usually fail to realize its importance unless it is diminished or reduced gradually. According to WHO (2015) 6.3\% of Indians are suffering from significant auditory loss. ${ }^{1}$ According to a study being conducted by Varshney (2016), ${ }^{1}$ in India 4 in every 1000 children suffer from severe to profound hearing loss. ${ }^{1}$ The impact of delayed diagnosis and proper intervention in infants with hearing loss has a severe negative effect on the overall speech and language, as well as the cognitive and social skills. ${ }^{2,3}$ However there are studies being conducted which states that these negative consequences can be reduced to some extent or prevented through early identification and intervention. ${ }^{4,5}$ Medical students working with general practitioners, otorhinolaryngologists (ENTs), pediatricians, neurologist, obstetricians/gynecologists, speech language pathologist and audiologists, are often involved together in the hearing testing of newborns and children. Medical students frequently work together in various setups such as government and corporate hospitals, primary health centers, medical college, camps, clinics, and private institutes. Medical students may play an important role in guiding patients and their families through diagnostic and treatment procedures. Similarly, medical students should also be aware about vestibular disorders as patients with vestibular disorders visits neurologist, otolaryngologist, general physician and audiologist. ${ }^{6}$ Knowledge about vestibular disorders helps the medical students in guiding patients about evaluation and management by concerned professionals. Vestibular disorders usually present with vertigo, a subjective symptom characterized by altered motion perception. Patients with hearing and vestibular disorders often visit medical colleges, government hospitals, primary health centers Medical students may play an important role in guiding patients and their families through diagnostic and treatment procedures of hearing and balance disorders. Yerraguntla et al. in $2016^{6}$ investigated knowledge and attitude of medical intern in coastal Karnataka, showed positive attitude toward different hearing related issues. ${ }^{6}$ There is a dearth of literature regarding attitude and knowledge about hearing impairment and balance disorders among medical students of North India. The present study aims to investigate knowledge and attitude toward hearing and balance disorders among medical students.

\section{Materials and Methods Participants}

A total of 266 medical students from government and private medical colleges were recruited in the present study. Informed consent were taken from all participants. All the participants were aware about the purpose of study. All the participants were native speaker of Hindi having good knowledge of English.

\section{Questionnaire}

A questionnaire-based study (online survey) of 22 questions was designed and adopted from similar studies to assess the knowledge and attitude towards hearing impairment and vestibular disorders among medical students. ${ }^{6-8}$ The 
questions were closed set task with 5 point rating scale from strongly agree to strongly disagree (Strongly agree, agree, neutral, disagree and strongly disagree). The questions used to assess knowledge and attitude towards hearing impairments includes 'Hearing loss is a condition that can become extremely disabling', 'hearing aid can be fitted at any age', 'importance of speech language therapy for children using hearing aids', 'learning to read and to write depends more on hearing than sight', 'hearing is an important sense in the development of speech and language of the child', 'importance of new born hearing screening', 'reservation in job and education for individuals with hearing impairment', 'hearing and speech developmental milestone play significant role in child's overall development', 'effect of hearing and speech disorder on social life of the patient', 'listening music at higher volume can cause hearing', ' Consanguinity (marriage in blood relation) can cause hearing loss', 'if a person is feeling ringing/buzzing sound in the ear, which professional he/she should contact'. Few question with 2 points rating scale includes 'Is it possible for young people like you to lose their hearing' and '.Have you ever heard the course like Speech and Hearing or Audiology and Speech Language Pathology. A open set question 'Which professional are responsible for hearing assessment?' was also asked from medical students.

The questions used to assess knowledge and attitude towards vestibular disorders includes 'balance problem could be due to dysfunction in his/her inner ear', 'vertigo can occur with tinnitus', 'in case of vertigo, which professional to contact'.

\section{Data Analysis}

Data collected was statistically calculated using SPSS 20 (SPSS Inc., South Asian Ed, Bengaluru, Karnataka, India). Descriptive statistics was used to summarize the data and the scores thus, obtained were cross-tabulated to obtain percentages.

\section{Results}

The data was analyzed and percentage of responses were calculated. Most of the medical students were agree of the fact that hearing loss can become extremely disabling condition. $52 \%$ of the medical students were not aware about the importance of hearing aids. 53\% of the medicos were unaware about the fact that hearing aid can be fitted at any age.

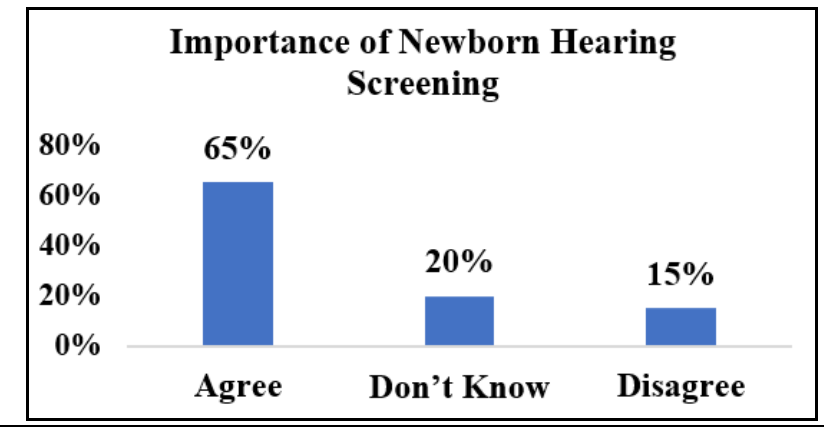

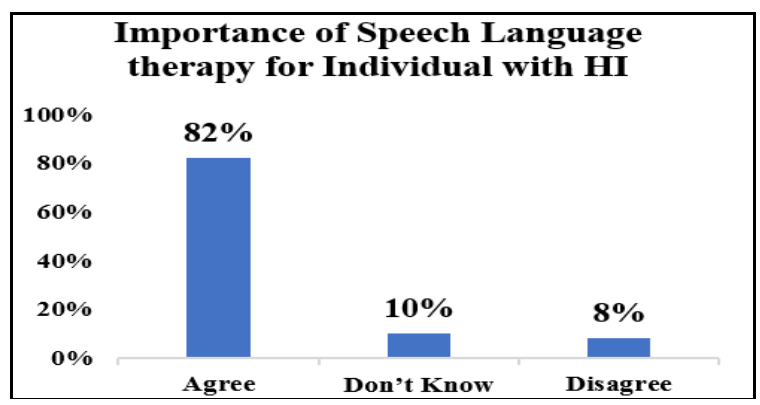

Fig. 1: Response for the question "importance of speech language therapy for the individuals with hearing impairment"

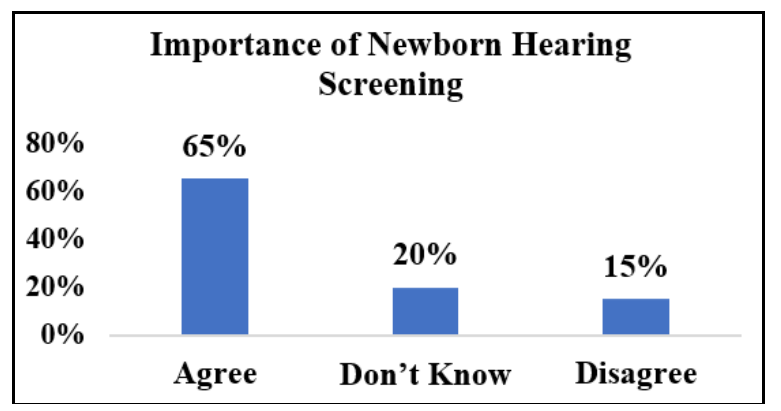

Fig. 2: Response for the question "Importance of New Born Hearing Screening"

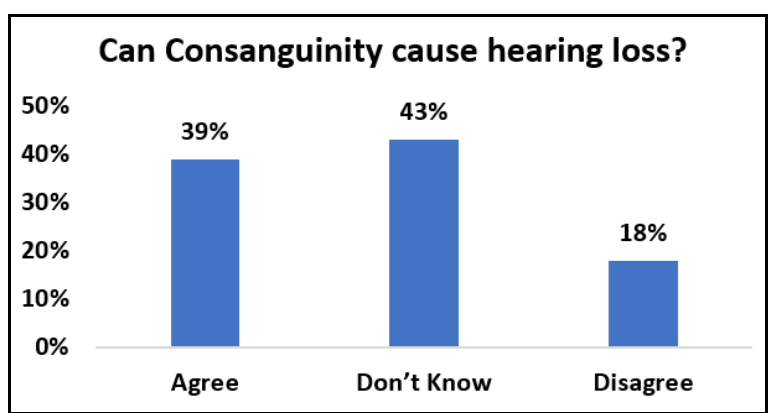

Fig. 3: Response for the question "Can Consanguinity cause hearing loss?"

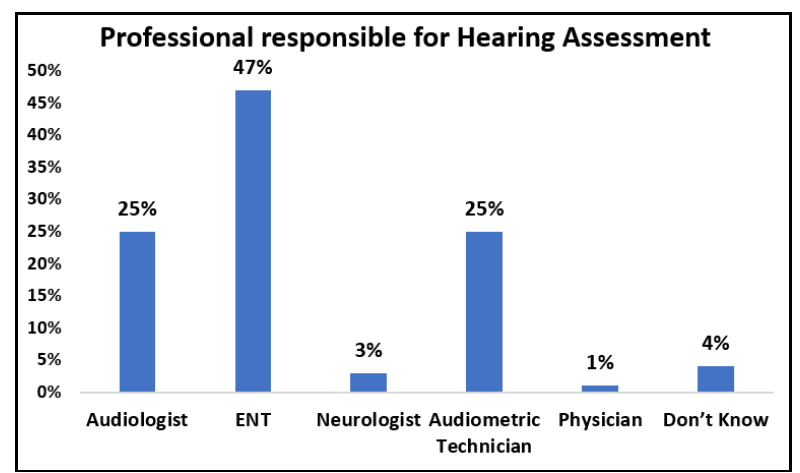

Fig. 4: Response for the question "Professional responsible for hearing assessment". 


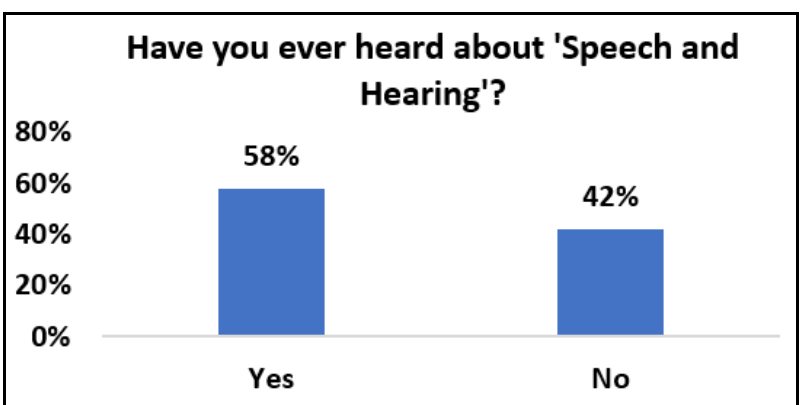

Fig. 5: Response for the question "Have you ever heard about speech and hearing?"

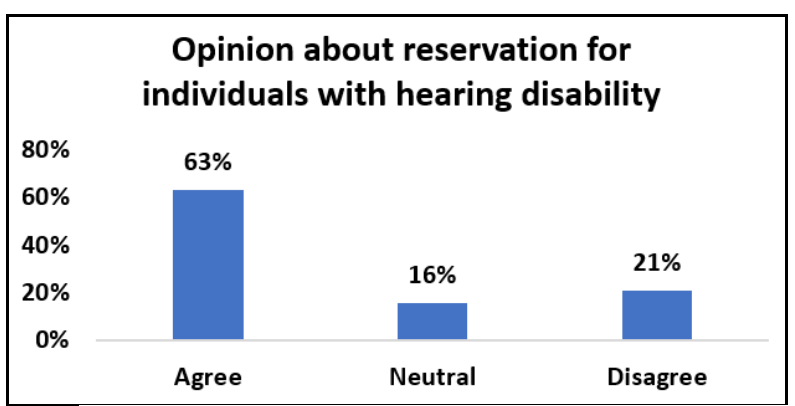

Fig. 6: Response for the question "opinion about reservation for individual with hearing disability"
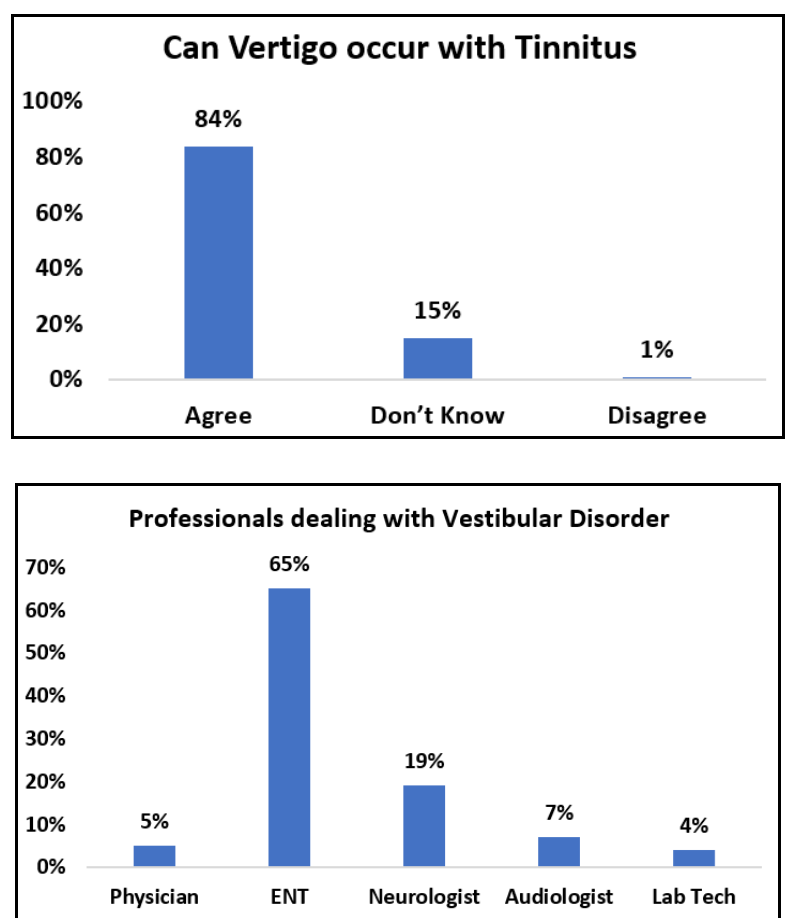

Fig. 7: Responses showing for the question been asked with respect to "whom to refer" for hearing loss

$81 \%$ of the medical students agreed the importance of speech language therapy for children with hearing impairment. $98 \%$ of the medical students thinks that hearing is an important sense to learn speech and language. $13 \%$ of the students deny the importance of newborn hearing screening. $22 \%$ were not knowing the importance of new born hearing screening; whereas, $65 \%$ of the medical students agreed for the importance of new born hearing screening. $96 \%$ of the medicos considered hearing and speech developmental milestone play significant role in child's overall development. Only $63 \%$ of the medical students supported reservation for the individuals with hearing impairment. More than $90 \%$ of medical students considered adverse effect of hearing and speech disorder on social life of the patient. From the present study it can be observed that $98 \%$ of the medical students were aware of the harmful consequences of listening music at higher volume.

$43 \%$ of the medical students were not knowing that consanguinity (marriage in blood relation) can cause hearing loss, whereas, $16 \%$ disagreed for the same. $92 \%$ of the medical students suggested to meet otolaryngologist in case of hearing loss and only 5\% suggested to meet audiologist. $94 \%$ of the medical students agreed the fact that even young people can lose their hearing. $83 \%$ of the medical students suggested to consult an otolaryngologist in case of buzzing sound in the ear. $41 \%$ of the medical students were unaware about the courses like 'Speech and Hearing' and 'Audiology and Speech Language Pathology'.

$90 \%$ of the medical students were aware of the fact that balance problem could be due to dysfunction of the inner ear. In case of vertigo, $65 \%$ and $18 \%$ of the medical students suggested to visit otolaryngologist and neurologist and only $6 \%$ suggested to visit an audiologist. $84 \%$ of the students reported that vertigo can occur with tinnitus.

\section{Discussion}

As the study shows that more than $50 \%$ of the students are not aware that an audiologist play a crucial role in managing patients with either hearing loss or vestibular disorder. They also tend to show poor knowledge regarding the risk factors responsible for causing hearing loss. Similar kind of studies have been found, in which the results reveal that more than $75 \%$ of the students tend to have poor knowledge compared to physicians about risk factors for hearing loss. But on the other side they tend to reflect adequate knowledge regarding the importance of newborn screening. As, more than $90 \%$ agree to the saying that hearing screening is important for each and every infant before getting discharged from the hospital. Campos et al.,(2014) ${ }^{9}$ had done a study which shows that more that $68 \%$ person of the health care professionals in which medical students also participated had lack of knowledge regarding the various assessment test available for pediatric hearing loss but showed good response with respect to age that the child can receive auditory rehabilitation and hearing screening programs. It was very surprising to see that in spite of having good knowledge regarding hearing loss more than $90 \%$ students didn't know that a person suffering from balance disorder can be referred to an audiologist which clearly symbolizes the fact that there is al lack of awareness regarding the role of an audiologist among the medical students. For the question on the importance of hearing screening most of the participants showed a positive attitude. Similar kind of 
findings were found in a study conducted by Danhauer et al. $2006^{9}$ wherein more than $80 \%$ professionals were aware that hearing loss could be identified by 3 months of age and strongly agreed to the implementation of the hearing screening.

\section{Summary and Conclusion}

In the present study, even though medical students showed adequate knowledge and positive attitude toward hearing impairment and vestibular disorders, there are certain areas which needs attention and concern to develop awareness among medical students. There is a need to educate these professionals regarding role of audiologists and speech language pathologist in hearing and balance disorders. Importance of newborn hearing screening and role of amplification device in management of individuals with hearing impairment need to be focused, while educating these professionals.

\section{Conflict of Interest: None.}

\section{References}

1. Varshney S. Deafness in India. Indian J Otol 2016;22:73.

2. White KR, Forsman I, Eichwald J, Munoz K. The evolution of early hearing detection and intervention programs in the United States. In Seminars in Perinatal 2010;34:170-9.

3. Yoshinaga- Itano C. Early intervention after universal neonatal hearing screening: impact on outcomes. Dev Disabilities Res Rev 2003;9:252-66.
4. Yoshinaga-Itano C. Levels of evidence: Universal newborn hearing screening (UNHS) and early hearing detection and intervention systems (EHDI). J Commun Disord 2004;37:451-65.

5. Kennedy C, McCann D, Campbell MJ, Kimm L, Thornton R. Universal newborn screening for permanent childhood hearing impairment: An 8-year follow-up of a controlled trial. Lancet 2005;366:660-2.

6. Yerraguntla K, Ravi R, Gore S. Knowledge and attitude of pediatric hearing impairment among general physicians and medical interns in coastal Karnataka, India. Indian J Otol 2016;22:183.

7. R. Ravi, D.R. Gunjawate, K. Yerraguntla, L.E. Lewis, B Rajashekhar. A national survey of knowledge, attitude and practices among pediatricians towards newborn hearing screening in India. Int J Pediatr Otorhinolaryngol 2017;95:9-14.

8. Goedert MH, Moeller MP, White KR. Midwives' knowledge, attitudes, and practices related to newborn hearing screening. $J$ Midwifery Women's Health 2011;56:147-53.

9. Danhauer JL, Johnson CE, Finnegan D, Lamb M, Lopez IP, Meuel C, et al. A national survey of pediatric otolaryngologists and early hearing detection and intervention programs. $J$ Am Acad Audiol 2006;17:708-21.

How to cite this article: Sanju HK, Aggarwal K, Yadav AK. Knowledge and attitude toward hearing impairment and vestibular disorders among medical students of north India. Indian J Anat Surg Head Neck Brain 2019;5(2):40-3. 